\title{
Interface de Usuários para o Enriquecimento da Consciência Situacional em Sistemas de Gerenciamento de Emergência
}

\author{
Natália Oliveira, Centro Universitário Eurípides de Marília - UNIVEM; Fábio Rodrigues, Centro Universitário \\ Eurípides de Marília - UNIVEM; Jessica Souza, Universidade Estadual Paulista - UNESP; Leonardo Botega, Centro \\ Universitário Eurípides de Marília - UNIVEM; Regina Borges de Araújo, Universidade Federal de São Carlos - UFSCar
}

\begin{abstract}
Resumo - Consciência Situacional (SAW) é um conceito amplamente utilizado em áreas que demandam tomadas de decisão crítica, como no domínio de gerenciamento de emergências. SAW está relacionada com o nível de percepção e entendimento que um indivíduo tem sobre eventos reais que ocorrem em cenários complexos, os quais devem ser geridos por sistemas críticos. Tais sistemas críticos exigem interfaces de usuário (UI) especializadas para fornecer aos operadores uma compreensão dinâmica do que está acontecendo no ambiente. Uma questão desafiadora no design de interfaces orientadas à SAW, é determinar como o processo de interface humano-computador pode ser construído para o enriquecimento de SAW, considerando ambientes com fontes de dados heterogêneas, limitações quanto à qualidade de dados e situações em constante mudança. $O$ problema aumenta quando informações estão sujeitas a incertezas, o que pode comprometer o processo de análise de situações. Além disso, os humanos tomam decisões baseadas em sua própria compreensão sobre o evento, o que aliado à experiência e conhecimentos podem ser ativos valiosos para serem usados para processar informação situacional sobre emergências para a aquisição de SAW. O objetivo deste trabalho é demonstrar como incluir uma UI orientada a SAW no processo de avaliação de situações de emergências e apresentar o desenvolvimento de uma UI que promova o gerenciamento de informação situacional de emergências para promover a aquisição de SAW. Os resultados apresentam uma rotina especificada para empregar UIs especializadas em SAW como parte de um processo de avaliação de situações, que suporta uma forte integração entre o humano operador e outras fases do processo, como a avaliação da qualidade, fusão de dados e visualização da informação, bem como um protótipo de interface que atenda ao processo. Um estudo de caso com um cenário crítico de um evento de roubo é também apresentado para demonstrar a aplicabilidade da abordagem proposta.
\end{abstract}

Palavras-Chave - Consciência Situacional; Interface de Usuários; Avaliação da Situação

Abstract - Situation Awareness (SAW) is a concept broadly employed in areas that require critical decision making, such as the emergency management domain.
SAW is related to the perception and the understanding level that an individual have about real life events that occurs in complex a scenarios, which must be managed by critical systems. Such critical systems require specialized user interfaces (UI) to provide to operators a dynamic comprehension about what is going on at the environment. A challenge issue regarding SAW-oriented interface design is to determine how the human-computer interfacing process can be built to enrich SAW considering environments with heterogeneous data sources, data with quality limitations and changing situations. The problem increases when information are subject to uncertainty, which can compromise the situation analysis process. Additionally, humans make decisions based on their comprehension about the event, which allied with the experience and knowledge can be valuable assets to be used to process emergency situational information for SAW acquisition. The objective of this work is demonstrate how to include SAW-oriented UIs into the process of emergency situation assessment and to present the development of a UI that promotes the management of emergency situational information to promote the SAW acquisition. The results present a specified routine for the employment of SAW-Specialized UIs as part of a situation assessment process, that support a strong integration between the human operator and others phases of the process, such as the quality assessment, data fusion and information visualization, as well as an interface prototype that supports the process. A case study with a critical scenario of robbery event is also depicted to demonstrate the applicability of the proposed approach.

Index Terms- Situational Awareness, User Interfaces, Situation Assessment

\section{INTRODUÇÃO}

C onsiência Situacional (SAW) é uma rotina baseada no desenvolvimento da percepção e compreensão do que está acontecendo dentro de um volume e espaço de tempo, e também a antecipação de eventos futuros.

SAW é visto como um fator crítico para o sucesso de todos os sistemas de apoio à decisão [1] e também é amplamente reconhecido como fornecedor base para a boa tomada e decisão. Em sistemas de avaliação de situações, 
tomadas de decisões assertivas são necessárias, principalmente em cenários críticos, desta forma a SAW deve ser analisada quanto ao nível de compreensão do especialista sobre determinado cenário.

Em cenários de tomadas de decisões críticas, como no domínio de chamadas de emergência, uma má SAW pode prejudicar a compreensão da situação, comprometendo a alocação de recursos e a preservação de patrimônios e vidas humanas.

A melhoria no processo de SAW pode auxiliar processos operacionais, auxiliando no planejamento de ações estratégicas, melhorando a qualidade das decisões e permitindo melhor compreensão de todo um cenário.

Um dos processos essenciais para dar suporte à análise de situações e consequentemente promover SAW é o de representação e controle da informação.

Representar informações inferidas do mundo real para a tomada de decisão militar apresenta desafios, pois tais ambientes possuem informações de natureza dinâmica e complexa [2].

Uma questão desafiadora na comunidade de avaliação de situações é determinar como o processo de inferência e representação da informação situacional pode ser redesenhada para o enriquecimento de SAW, que pode ser severamente degradada se a informações de baixa qualidade são produzidas e propagadas por todo o processo [3].

Além disso, fornecer aos seres humanos dados e informações sobre a qualidade das informações, para que adquiram uma melhor compreensão do que está acontecendo no ambiente, são fatores que auxiliam o processo de tomada de decisões [2].

Neste artigo, será apresentado o desenvolvimento de uma interface de usuários dedicada a representar e gerir a informação produzida por um sistema de avaliação de situações.

Uma combinação de técnicas e ferramentas com o objetivo de apresentar informações semânticas qualitativas, através de uma interface de usuário, buscará auxiliar que a melhoria da percepção das situações com a estrutura proposta por um processo para que de forma madura auxilie o processo de tomada de decisão em cenários de roubo, visando à redução da agregação de erros humanos que comprometam SAW em sistemas de avaliação de situações.

Um estudo de caso no contexto de análise de informações incertas para a consciência situacional de eventos de crime é descrito. Neste cenário, a interface e visualizações em sinergia com as informações incertas propagadas no processo, são avaliadas quanto à contribuição para o processo de SA e consequentemente a obtenção e manutenção de SAW.

$\mathrm{Na}$ Seção 2 serão apresentados conceitos de SAW e avaliação da situação, tal como a sua relação e consequência. A Seção 3 aborda um estudo sobre a qualidade de dados no processo de SAW. Na Seção 4 a qualidade da informação e a incerteza na avaliação de situações no domínio de SAW são apresentadas. Na Seção 5é abordada à qualidade de dados e sua importância em sistemas de gerenciamento de emergências... A Seção 6, interfaces orientadas a SAW são mencionadas, bem como a apresentação de trabalhos relacionados. A Seção 7 uma proposta para interfaces orietadas à SAW em sistemas de avaliação de emergências é proposta, e finalmente, na Seção 8 o desenvolvimento ds interface é descrito.

\section{SAW E A INFLUÊNCIA NA AVALIAÇÃo DE SituAÇÕES}

Consciência da situação (SAW) consiste em estar ciente dos eventos que ocorrem em um ambiente e compreender como essa informação afeta a situação no presente e no futuro próximo.

De acordo com Endsley em [4], atingir SAW é um processo realizado pelo usuário, e é composto por três níveis: percepção dos elementos no ambiente, compreensão da situação atual e sua projeção um estado futuro. $\mathrm{O}$ primeiro nível lida com a percepção dos elementos dos ambientes, após alcançar tal percepção, o próximo passo para alcançar SAW consiste na compreensão dos dados e a relação destes com as metas e objetivos do operador. Após compreender os elementos e a relação destes com tal objetivo, o operador consegue deduzir em curto prazo o estado destes elementos no futuro, o que corresponde ao nível três alcançando assim SAW. A literatura descreve oito grandes fatores que podem atrapalhar o processo de SAW. Esses oito fatores são: gargalo de atenção, limitação da memória, estresse, excesso de dados, saliências mal inseridas, complexidade do sistema, modelos mentais incorretos e automação.

1) Gargalo de atenção: relaciona-se com a incapacidade do ser humano de prestar atenção a mais de uma informação por vez, dificultando o processo de SAW em vista da grande quantidade de informações expostas por sistemas que visam auxiliar este processo;

2) Limitação da memória: em curto prazo é utilizada para formar uma figura mental do que está acontecendo por meio da experiência do usuário e conhecimento armazenados na memória em longo prazo, essa memória em curto prazo, no entanto, é limitada conduzindo a implicações no processo de SAW;

3) Fatores de estresse: como a carga de trabalho, ansiedade, fadiga que podem sobrecarregar e prejudicar seu processo de cognição;

4) Excesso de dados: refere-se à rápida mudança de dados dificultando os operadores de manter-se atualizados e, consequentemente minar o processo de SAW;

5) Saliências mal inseridas: consistem em informações mal colocadas ou mal destacadas. Destacar informações pode ser de grande ajuda no processo de 
SAW, porém, caso mal inseridas conduz o usuário a trilhar caminhos para tomada de decisão baseados em informações irrelevantes ao contexto;

6) Complexidade do sistema no qual muitas funcionalidades criam uma barreira para que o usuário desenvolva um modelo mental de como este funciona

7) Modelos mentais incorretos: gerados com base em situações similares ao usuário, mas que não se encaixam diante do contexto atual conduzindo a uma má interpretação da situação.

8) Automação que pode levar a dependência do usuário em relação ao sistema [4]. Dentre incidentes relacionados à compreensão da situação em razão de uma má SAW, a maioria deles classificam-se entre os oito fatores que dificultam o processo de cognição da SAW, dentre oitos fatores três se relacionam com a utilização de sistemas como o excesso de dados, saliências mal inseridas e a complexidade do sistema em questão.

Endsley [5] apresenta uma taxonomia de erros em SAW de maneira a descrever muitos fatores que podem levar a erros neste processo. Tal taxonomia foi aplicada por meio de uma investigação nas principais transportadoras aéreas nos Estados Unidos [6]. De acordo com os resultados $88 \%$ dos erros envolveram SAW, estes foram divididos entre os três níveis de SAW tendo como causas variadas, dentre elas: dificuldade de detectar informação, falha ao monitorar, criação de um pobre modelo mental, exagero ao projetar possíveis situações.

Rodgers em [7] relatam que dentre os grandes fatores que conduziram a erros operacionais em terminais de controle de tráfego aéreo em 1997 nos Estados Unidos, $58 \%$ foram associados com problemas relacionados à SAW. Como registrado, a compreensão das situações e como componentes desta impactam o ambiente é um fator crucial para a tomada de decisão e influencia o comportamento do próprio usuário e sistemas que auxiliam a conclusão de tal processo onde casos mal sucedidos, dependendo do domínio da aplicação, podem conduzir a erros irreversíveis. Assim, fica evidente a importância de levar em consideração os níveis de SAW, bem como evidenciá-los no desenvolvimento de sistema de avaliação de situações.

\section{QUALIDADE DE DADOS E INFORMAÇÕES}

A necessidade de representação gráfica e controle de informações para auxílio ao processo de tomada de decisão militar apresentam desafios, devido à necessidade de fornecer aos tomadores especialistas de decisão, subsídios para a uma compreensão da realidade de um cenário em tempo real [8].

Entretanto, informações com problemas de qualidade podem prejudicar o modelo mental do usuário, visto que tais informações descrevem a realidade do cenário de acordo com os contextos e podem conduzir a incertezas ao operador ao tentar interpretar os dados.

Em [9] os autores, descrevem incerteza como uma questão generalização que engloba conceitos, como inconsistência, dúvida, confiabilidade, imprecisão e erro, podendo incluir ainda variações estatísticas, diferenças, ruídos ou dados faltantes.

Entretanto, há um consenso de que se a incerteza existe e é conhecida, a mesma deve ser visualizada e transmitida ao especialista.

Assim, a análise de informações tem por objetivo não apenas avaliar informações disponíveis e a forma com as quais são organizadas, mas também deve ajudar a orientar o especialista a raciocinar sob incertezas [8].

A qualidade dos dados pode ser definida como um dos fatores cruciais para sistemas de tomada de decisão. Informações imperfeitas, que não descrevem fielmente as situações do mundo do real diminuem a efetividade dos sistemas, contribuem de forma negativa para a formação do modelo mental de usuários e, consequentemente minam o processo de SAW.

Em cenários de avaliação de situações, a qualidade de dados é um fator crítico essencial, visto que a informação situacional se propaga ao decorrer do processo, podendo ser comprometida em transformações no decorrer do tempo.

O processo de definição da representação das informações através de uma interface de usuário com apoio a aquisição de SAW é um trabalho desafiador, desta forma, o objetivo desta pesquisa é permitir que a interface possa auxiliar o processo de aquisição, manutenção e melhoria da SAW na mente humana, através das características contidas na composição desta interface.

\section{QUALIDADE DA INFORMAÇÃo NA AVALIAÇÃo DE SITUAÇÕES}

Use De acordo com a literatura, não há um padrão definido quanto à qualidade da informação em sistemas de tomada de decisão. Os requisitos são divididos em dimensões ou métricas e a aplicação destas é altamente dependente de domínio, considerando que a aplicação define seus respectivos significados de acordo com objetivos, tarefas e decisões associadas.

Para o domínio de roubo, a metodologia a ser definida aborda avaliações de qualidade quanto à completude e aspectos temporais dos dados. As abordagens e descrições de diferentes perspectivas serão descritas a seguir.

O’Brien em [10] define as dimensões de qualidade necessárias para sistemas de informação em três dimensões principais divididas em: Conteúdo, Tempo e Forma. Dentre os atributos de qualidade constam:

1) Tempo: Prontidão, Aceitação, Frequência, Período.

2) Conteúdo: Precisão, Relevância, Integridade, Concisão, Amplitude, Desempenho.

3) Forma: Clareza, Detalhe, Ordem, Apresentação, Mídia. 
Em [11], Wang e Strong categorizam os atributos das dimensões da qualidade em quatro classes principais.

Qualidade Intrínseca de dados implica a garantia da credibilidade e reputação dos dados, dentre os atributos constam a própria credibilidade e reputação, como precisão e objetividade.

Qualidade de dados contextual é formada por atributos que devem ser considerados e avaliados de acordo com o contexto da tarefa a ser realizada, tendo como atributos: relevância, tempo, completude, etc.

Quanto à qualidade de representação, os atributos são definidos de acordo com aspectos relacionados ao formato do dado (como a concisão e representação) e o significado em relação a compreensão e interpretação de tais dados. Por fim, os autores classificam individualmente os atributos relacionados à acessibilidade.

Carlo Batini em [12] afirmam existir muitas discrepâncias quanto à definição da maioria das dimensões devido à dependência contextual da qualidade. Confirmam que não há uma definição global de um conjunto específico de dimensões que definem a qualidade dos dados.

Os autores em [12] abordam quatro dimensões, sendo estas: Precisão, Completude, Consistência e aspecto temporal do dado (Volatilidade, Atualização). Adiante seguem devidas descrições:

A precisão de um dado é medida por meio de sua equivalência com o dado em questão, assim, determinada proximidade de um valor $\mathrm{x}$ em relação a outro valor $\mathrm{x}$ pode ou não ser considerado preciso mediante o contexto no qual o dado está sendo testado.

Completude pode ser definida como o grau no qual o dado em questão abrange sua correspondente situação no ambiente real. Conforme abordado na área de pesquisas de bancos de dados relacionais completude relaciona-se com o significado e a representação de valores nulos.

A dimensão da consistência refere-se à violação de regras definidas ao determinado conjunto de dados, como por exemplo, se a idade do usuário for menor que 18 anos este não está apto para possuir a Carteira Nacional de Habilitação.

Dimensões relacionadas ao tempo, ou Timeliness, podem relacionar-se ao atraso na atualização dos dados entre seu estado no ambiente real e no sistema de informação. Bem como a idade meia do dado em sua fonte.

\section{QUALIDADE DE DADOS E INFORMAÇÕES NO GERENCIAMENTO DE EMERGÊNCIAS}

A qualidade dos dados em sistemas de avaliação de situações de emergências é um dos fatores críticos no processo de tomada de decisões, visto que o processo de consciência da situação pode ser prejudicado, tendo sua efetividade reduzida quando informações imperfeitas são inseridas ao processo, contribuindo negativamente a formação do mapa mental do operador para responder os chamados de emergência.

Sistemas de avaliação de situações costumam envolver esforços para estimular SAW nos operadores, pois tratam grandes quantidades e variedades de dados. Desta forma, o processamento e representação destas informações visam encontrar dados suficientemente e significativamente relevantes para auxiliar o processo de tomada de decisão.

Problemas referentes à qualidade da informação são elementos que estão associados à formação e composição de dados e informações, e que podem surgir desde o processo de aquisição dos dados até o seu processamento e cognição e representação da informação, um destes problemas é a incerteza.

Em [9] Pang, Wittenbrink e Lodha, descrevem incerteza como uma questão complexa que engloba conceitos, como inconsistência, dúvida, Confiabilidade, imprecisão e erro, podendo incluir ainda variações estatísticas, erros, diferenças, ruídos ou dados faltantes.

A incerteza é um elemento que está associado à formação e composição de dados e informações, e que pode surgir desde o processo de aquisição dos dados até o seu processamento e cognição.

$\mathrm{Na}$ tomada de decisão, particularmente na área de gerenciamento de emergência, o processo pode ser comprometido devido à decisão imprecisa originada da má interpretação dos dados.

A qualidade de dados e informações em sistemas de controle e gestão a eventos de emergência, por exemplo, pode fazer uso das dimensões de completude, precisão, atualidade e consistência, cada qual com formas particulares de ser calculada.

\section{A. A Representação Gráfica da Incerteza}

Sempre que os dados são processados, transformados e mapeados em representações visuais, a incerteza pode ser agravada, o que torna difícil manter a qualidade do dado ao longo do processo [13].

Com a visualização da incerteza disposta de forma explicita em todo o processo, o tomador de decisões terá acesso aos níveis de confiança de dados, proporcionando ao operador, informações obtidas, que possam auxiliar a tomada de decisão.

Adicionalmente, dimensões de qualidade de dados como precisão, integridade, consistência, dimensões de tempo [14] e taxonomias das causas do conhecimento imperfeito como as descritas por Gershon em [15], que envolvem dados e informações corrompidas, incompletas, inconsistentes, que causam dificuldade na compreensão e incerteza.

Representações de informações de um determinado objeto tais como incerteza, imperfeição e outras, podem ser abordadas de duas maneiras: intrínseca ou extrínseca [15]. As Intrínsecas são utilizadas para representar algo que faz parte do objeto, como variações na aparência. Já as extrínsecas, são utilizadas para mostrar o que não faz parte do objeto, apresentando novas estruturas associadas para identificar novos atributos [16].

Visto que a tomada de decisão militar é baseada em dados incertos, faz-se necessário tratá-la na visualização. Em [17], os autores apresentam uma classificação para técnicas de representação de incerteza: utilização de glifos, adição e modificação da geometria, de atributos, animação, sonorização e técnicas psicos-visuais, são técnicas que permitem maior cognição por parte dos utilizadores. As técnicas para 
representação de incerteza estudada em [18], não se limitam a um domínio específico, as aplicando em C2, objetiva-se obter facilidade na compreensão e apresentação das informações. As técnicas descritas são:

1) Variáveis gráficas livres: cor, tamanho, posição, foco, clareza, imprecisão, saturação, transparência e borda.

2) Objetos adicionais: rótulos, imagens ou símbolos. Incluindo a incerteza na magnitude, direção e comprimento em hieróglifos [17].

3) Animação: a incerteza é mapeada para parâmetros de animação como a velocidade ou duração, borrão de movimento, alcance ou extensão do movimento.

4) Representação interativa: por exemplo, incerteza pode ser descoberta pela interação do mouse [17].

5) Sonificação e técnicas psico-visual: incorporação de acústica, mudanças de tom, volume, ritmo, vibração ou piscar mensagens textuais [17].

Muito embora haja uma necessidade de visualizar incertezas associadas aos dados, a comunidade científica tende a separar a apresentação da incerteza do dado. Incluir informações de incertezas adicionais em um sistema de visualização existente é um processo trabalhoso e há uma ausência de métodos que o implementem.

A visualização de incertezas como suporte ao processo de tomada de decisão é um problema recorrente e interdisciplinar, e poucos estudos foram conduzidos para determinar o impacto da visualização de incertezas em usuários [19].

\section{B. Gestão da Incerteza em Sistemas de Avaliação de Situações e informações}

Não foi encontrado na literatura um padrão que especifique os processos de gestão da qualidade das informações para sistemas de avaliação de situações de emergência. A aplicação deste se deve ao cenário, onde são determinados os requisitos de qualidade (dimensões) para cada área [20].

Em [12], Batini define o gerenciamento da qualidade de dados e informações como a definição de procedimentos relacionados ao manuseio das informações, definindo um processo para medir, representar e melhorar a qualidade de dados e informações.

Cada domínio de aplicação estabelece seu próprio conjunto de dimensões e metodologias para quantificar e/ou qualificar cada dimensão, à luz dos requisitos informacionais da área específica. Adicionalmente, o domínio de aplicação das dimensões também influencia na forma com que cada uma delas é calculada, determinando as métricas de qualidade.

\section{INTERFACES PARA SAW}

Para dar suporte à $\mathrm{SAW}$, sistemas de apoio à decisão no domínio militar geralmente utilizam Interfaces de Usuário, do inglês User Interface (UI) que processam visualização de informações, como forma de apresentar produtos e subprodutos de etapas de sistemas de avaliação de situações. A cada conjunto de informações produzido, o especialista observa e se orienta à luz das informações representadas, para depois tomar uma decisão e agir de acordo com seu nível de SAW adquirido.
Interfaces de usuário também podem influenciar SAW. No contexto de obtenção e manutenção de SAW para o gerenciamento de emergências, a interface não é apenas o meio principal de representarão de informação, mas sim o passo inicial para estimular consciência da situação.

Uma questão desafiadora no design de interfaces orientadas à SAW é a forma como o processo de interação humanocomputador pode ser redesenhado para o enriquecimento de SAW considerando ambientes com potenciais de grande escala e fontes de dados heterogêneas com constante mudança em situações complexas. O problema aumenta quando tais informações estão sujeitas a incerteza, o que pode comprometer a aquisição da consciência situacional.

SAW orientada a UI para sistemas críticos exigem interfaces de usuário especializadas para fornecer aos operadores uma compreensão dinâmica do que está acontecendo em um ambiente.

A informação a ser gerenciada por essas interfaces afeta a forma como os operadores de um sistema de gerenciamento de emergência adquirem, mantém e recuperam SAW. Uma questão desafiadora no design de interfaces orientadas à SAW é a forma como o processo de interação humano-computador pode ser redesenhado para o enriquecimento de SAW considerando ambientes com potenciais de grande escala e fontes de dados heterogêneas com constante mudança em situações complexas.

Quando humanos e sistemas interagem para a avaliação das situações em um processo de inferência, SAW pode ser melhor adquirida, mantida e até mesmo restaurada. Muitas interfaces orientadas à SAW descrevem o papel humano de uma forma semi-automatizada.

As três principais visualizações do usuário aparecem para enriquecer a figura final com ou sem feedback intermediários: os seres humanos não apenas como consumidores, mas também produtores de informação e humanos como atores na informação [21] [12]. Tais interfaces são carentes de melhor investigação sobre as implicações e questões relacionadas à intervenção humana para construir e manter SAW.

As abordagens mais recentes apresentam oportunidades para interação humana em cada nível de avaliação para o enriquecimento de SAW [8]. Entretanto, apesar de tais interfaces incluírem o humano como ator no processo de avaliação da situação, as mesmas não fornecem acesso à informação em todo o processo de manipulação de fontes de informação.

No domínio de gerenciamento de emergência, a interface está presente onde um operador humano observa, se orienta, decide o que fazer e, em seguida, tomar algumas medidas, que podem ser tanto informações de solicitação de refinamento ou tomada de decisão específica de domínio.

\section{A. Trabalhos Relacionados}

Esta seção apresenta o estado da arte para as interfaces orientadas à SAW. Soluções existentes tipicamente visam capacitar o operador e o sistema de intensificar a sua relação com a informação para construir uma representação mais viável de situações. Entre os trabalhos relacionados há estruturas de interação para SAW, interfaces especializadas e sistemas gerais que se baseiam em discurso humanoinformação. 
Em [22], Nwiabu discutiu UIs para sistemas de comando e controle (C2) para predição de formação de hidratos em oleodutos e gasodutos submarinos, conforme Fig. 1. A interface baseia-se nos resultados de uma análise hierárquica de tarefa que se decompõe de um cenário complexo de pequenas tarefas. A interface do usuário é capaz de reconfiguração automática de se adaptar à situação atual e reduzir o esforço mental do operador.

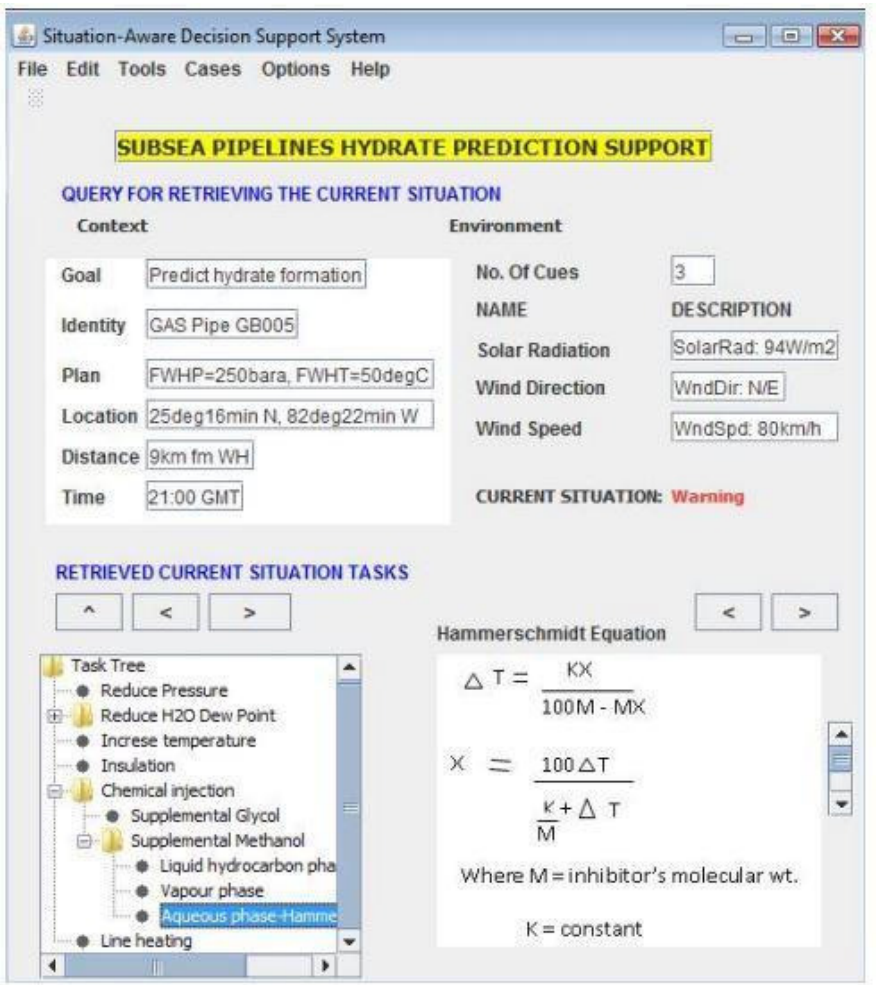

Fig. 1 - Interfaces de sistemas de C2 para predição de formação de hidratos em oleodutos e gasodutos [22].

Yu em [23] apresentou um novo contexto de visualização através da interface do usuário, que tem um motor de interpretação para as necessidades do operador, definir quais informações devem ser apresentadas. Para a melhoria da compreensão do operador, um mecanismo de controle impreciso foi proposto para realizar uma pesquisa difusa com base em palavras-chave específicas do domínio da aplicação por interações dos operadores.

Onal em [24] desenvolveram uma interface de usuário com base em métodos para melhorar SAW e também para minimizar o esforço mental de máquinas de mineração pesados, conforme mostrado na Fig. 2. O layout da interface do usuário é baseado na interação guiada, painéis de suporte, mapas virtuais e múltiplas telas. Tais componentes são integrados para ajudar os operadores a evitar acidentes devido à sobrecarga de informação em tarefas operacionais. A Análise de Tarefas Dirigidas a Metas (GDTA) foi aplicado para identificar requisitos.

Em [25], Chai e Du desenvolveram uma estrutura para suportar a aquisição SAW em um sistema de comando e controle (ataques no campo de batalha). Esse quadro baseia-se

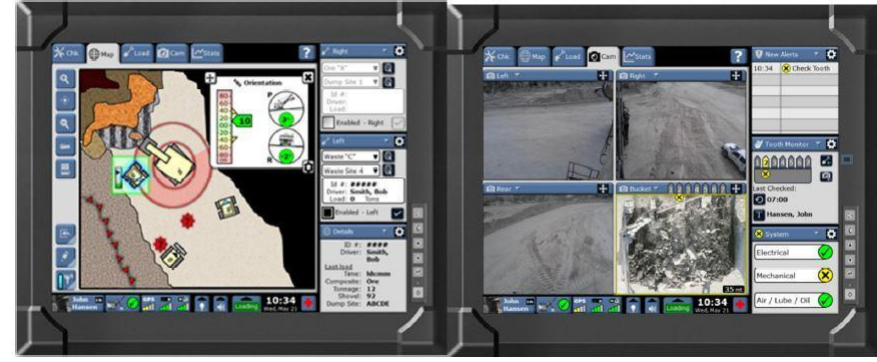

Fig. 2 - UI com base em métodos para melhorar SAW e minimizar o esforço mental do operador [24].

no uso de dados fundidos e regras de classificação para ajudar a reconhecer e explicar ativos inimigos e evolução, em um ambiente dinâmico.

Gomez em [26] criou um protótipo de interface para aumentar SAW dos tomadores de decisão durante o acompanhamento de jogos de futebol em tempo real. Seu desempenho permite uma oportuna alocação das equipes de resgate a incidentes respostas. Uma rede de sensores sem fio é responsável pela captura de dados heterogêneos e enviá-los para a interface do operador, que é inteiramente baseado em contexto temporal para representar o cenário. As visualizações incluem a localização do pessoal de salvamento e o local do acidente, dentro de um estádio.

Na Fig. 3 é mostrado que Feng em [27], desenvolveu um sistema de apoio à decisão que incorpora SAW compartilhada entre agentes que extraem a informação relevante sobre as entidades e representá-los para o operador. Esses agentes têm o seguinte conjunto de metas e estratégias para cada nível SAW: missões, planos, ações e atributos físicos. Em seguida, eles são responsáveis por gerar recomendações sobre o cenário. A interface do usuário lida com os aspectos espaçotemporais da evolução das missões. A interação limitada com as recomendações é permitida.

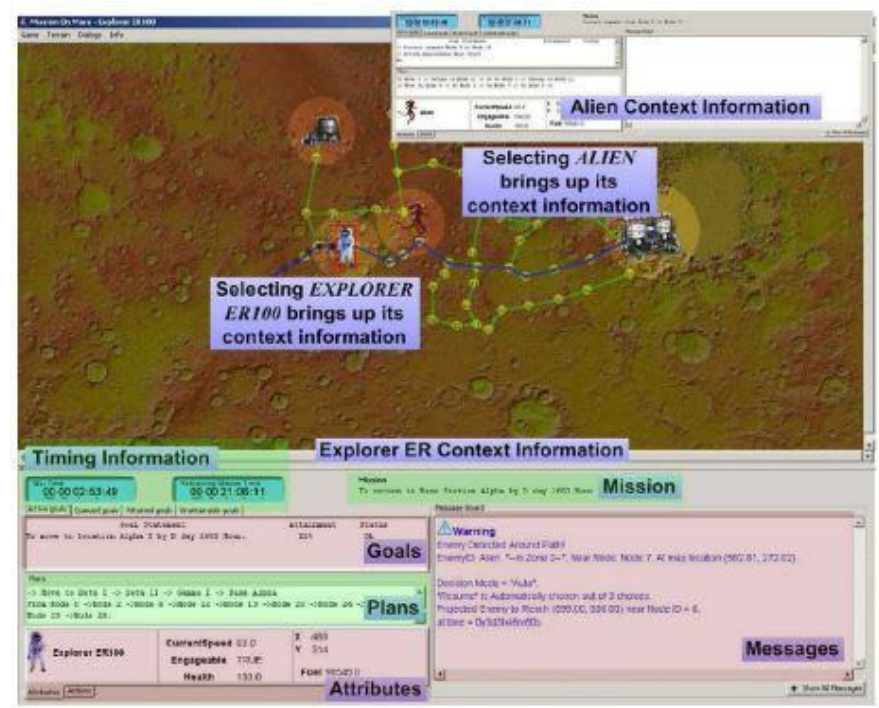

Fig. 3 - Interface de sistema de apoio a decisão que incorpora SAW compartilhada [27].

Além de serem soluções eficientes para os domínios de aplicações específicas, tais soluções são limitadas sobre a gestão da informação que está sendo propagada ao longo do ciclo de avaliação da situação. Nossa abordagem inova na 
promoção de um controle total da informação que é produzida em cada fase, usando representação incerteza e métodos de aperfeiçoamento como um recurso para controlar o conhecimento que é criado, representados e utilizados para avaliar situações.

\section{PROCESSO PARA O ENRIQUECIMENTO DE SAW NO CONTEXTO DE SITUAÇÕES DE EMERGÊNCIAS}

A estrutura conceitual para melhorar a consciência situacional para sistemas de gerenciamento de emergência e os seus principais componentes que descrevem como os processos de dedução, avaliação, representação visual das informações e sistemas humano de refinamento da informação podem ser explorados para apoiar SAW mediada por uma interface de usuário especializada estão representados na Fig. 4. Os componentes fundamentais são destacados (laranja).

O processo foi utilizado para desenvolver uma interface orientada à SAW para um relato de eventos roubo, como parte de um sistema de gerenciamento de emergência.

Antes de realizar qualquer análise de informação, o nosso quadro recebe a saída de uma fase de aquisição. Essa aquisição faz um Processamento de Língua Natural (PLN) para identificar objetos, atributos e propriedades de chamadas de áudio comunicados à Polícia Militar do Estado de São Paulo (PMESP). Como saída, é produzido um conjunto de objetos e propriedades que chamamos Situação. Tal situação é então submetida à Avaliação da Qualidade da Informação.

$\mathrm{Na}$ Avaliação da Qualidade da Informação, a situação produzida é submetida a uma análise das seguintes dimensões de qualidade: completude (relacionada com a presença ou ausência) de objetos ou atributos que os descrevem; currency (dimensão atualidade), que ajuda a determinar a "idade" da informação e assim tomar medidas oportunas; e incerteza, quando os operadores detêm apenas um conhecimento parcial sobre uma Situação em nosso domínio consideramos incerteza como uma generalização das outras dimensões. Situações são entidades que evoluem ao longo do tempo e devem ter sua qualidade da informação indexada e atualizada a cada vez que uma nova informação chega ou é produzida, e também o valor de sua incerteza.

O produto de aquisição e avaliação da qualidade é um conhecimento situacional em que tem de ser representada. Em nosso sistema de avaliação da situação completo, o modelo ontologia foi escolhido para representar a semântica da informação gerada, devido à flexibilidade em representar as relações entre os objetos. Nesta fase, já são conhecidos, os objetos, atributos e possíveis relações entre eles. Em sistemas de avaliação de situação, é chamado Nível 1 e Nível 2 da avaliação, também correspondente aos níveis de percepção e compreensão de uma percepção da situação [4]. É este conhecimento que deve ser codificado em visualizações e gerido pela UI.

Processo de aquisição gera entidades que serão codificadas na visualização. Para complementá-lo, integração de informação pode ser realizada utilizando os objetos já produzidos como entrada. Essa integração, conhecida como Fusão de Informações, é capaz de produzir em uma dimensionalidade menor, novos objetos e novas relações semânticas que também devem ser representados graficamente. O produto desta fase, chamado Situação, é

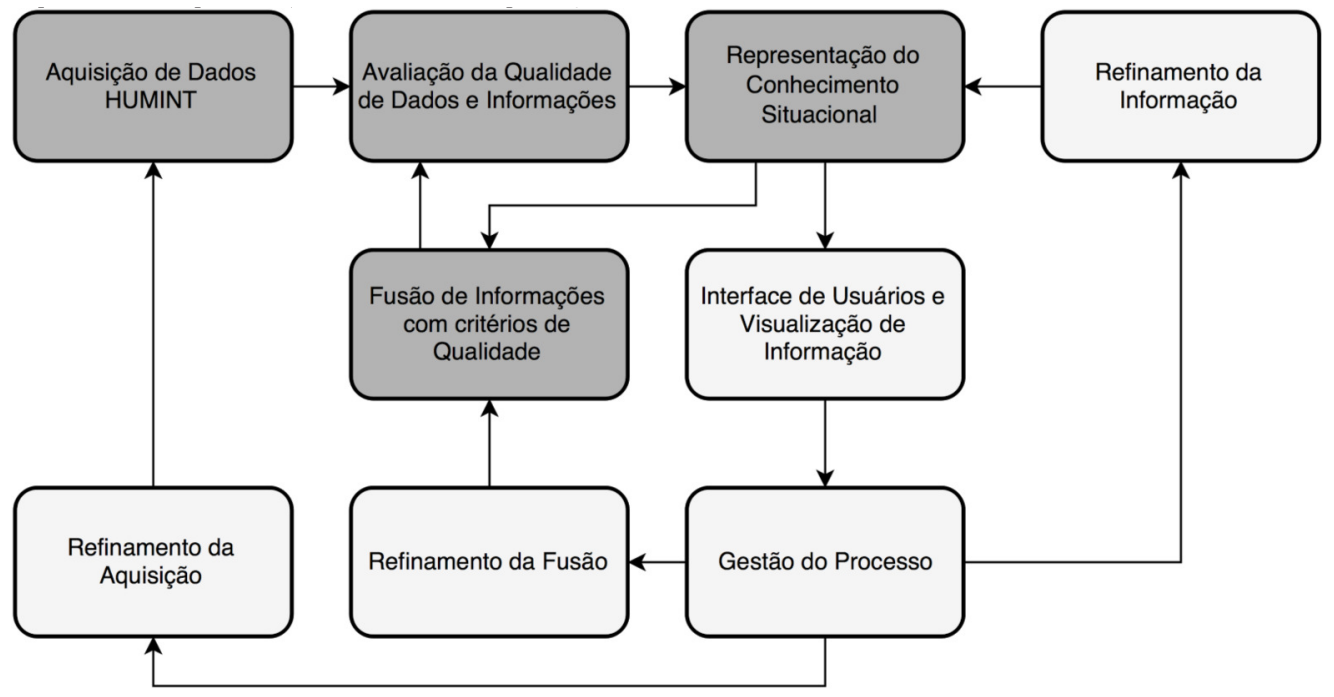

Fig. 4 - Apresentação do framework conceitual desenvolvido para descrever a interface do usuário no contexto da Avaliação da Situação.

submetido à avaliação da qualidade da informação, o que enriquece o conhecimento situacional existente e é codificado em visualizações posteriores.
O próprio UI detém o gerenciamento de informações de todo o processo. Nossa interface é uma bancada colaboração onde ambos, sistema e o operador fornecem e transmitem 
informações como um conhecimento parcial que evolui fora do tempo estipulado. Portanto, toda vez que uma nova informação é fornecida por um desses atores principais, o outro deve processar e reconstruí-lo como um novo conhecimento.

No domínio de gerenciamento de emergência, a interface está presente onde um operador humano observa, se orienta, decide o que fazer e, em seguida, tomar algumas medidas, que podem ser tanto informações de solicitação de refinamento ou tomada de decisão específica de domínio. Em nossa abordagem, este é o lugar onde o sistema compartilha o conhecimento parcial gerada pelas outras fases e, em seguida, escuta as entradas dos operadores de forma cíclica.

Realizada pela UI, as visualizações transmitem o conhecimento agregado sobre Situações. Existem dois métodos gráficos disponíveis para a análise do especialista: a visualização com base em mapa e o grafo das relações hierárquicas

O uso de sobreposições em um mapa geo-referenciado é um requisito para as operações do sistema de envio de emergência, sendo dependente de atributos de localização, crucial para determinar o comparecimento a um evento de emergência. Assim, os outros objetos que compõem uma situação complementar a visualização como informações sobre criminosos, vítimas e objetos roubados, por exemplo, cada um com sua própria descrição.

A adopção de estrutura de um grafo é justificada pela necessidade de conhecimento hierárquico sobre como a informação é construído sobre situações e seus objetos. É necessário que os operadores de polícia saibam como cada situação é composta, e com quais objetos e atributos. Essa hierarquia foi obtida através da análise de requisitos com a PMESP. Situação é a entidade central, composta pela relação entre objetos e seus atributos e ramificações. Objeto pode ser identificado e não têm relações em tudo, por isso, podem estar em hierarquias independentes. Neste caso, mesmo quando não está compondo uma situação, ele deve ser representado. Em nosso estudo de caso, a situação é um relatório de eventos roubo e seus objetos são vítimas, criminoso, localização e objetos roubados, cada um com um conjunto de características que chamamos atributos.

\section{INTERFACE DE USUÁRIOS ORIENTADA À SAW: CASO DE RELATO DE ROUBO}

Para o levantamento de requisitos do desenvolvimento da nossa UI, foram adotadas duas abordagens: GDTA e as diretrizes para projetar para a consciência da situação, tanto introduzido por Endsley [5]. O GDTA ajuda os designers para listar todas as informações necessárias para estimular cada um dos três níveis de serra (Percepção, Compreensão, de Projeção) e tarefas relacionadas para ajudar a obtê-los. Para adquirir tais informações-, um questionário e uma observação no local foram aplicados aos especialistas. Este levantamento também ajudou a definir as prioridades e as decisões que devem ser executadas durante a observação da informação.

Nesta seção são apresentados os princípios para o desenvolvimento de cada componente da nossa interface do usuário e as escolhas de design baseado no estado da arte.
Ressaltam-se também as vantagens e desvantagens de cada escolha de design para o nosso domínio.

\section{A. Organização das informações de acordo com os objetivos}

O objetivo é analisar e avaliar situações do domínio de gerenciamento de cenários complexos, mais especificamente, situações de emergências, para ajudar os humanos operadores a adquirir SAW usando as informações coletadas e processadas a partir do sistema de avaliação.

Para obter este resultado, a representação da informação foi estruturada em torno de objetivos, assim, é necessário que a interface não seja totalmente orientada por dados, visando a contribuição para SAW. Assim, a interface foi dividida em três visões diferentes, porém interconectadas. A figura 5 apresenta a interface de visualização principal para a aquisição de SAW em situações de emergência.

A primeira visão (canto inferior esquerdo) na interface do usuário é a tabela de um objeto para os eventos de entrada, contenção: fonte de informação, objetos encontrados por aquisição e fusão, acrescentou informação tempo e a qualidade da informação (incerteza total sobre objeto) avaliada.

Nesta tabela são especificadas todas as transformações que a situação é submetida, como por exemplo: a inserção de um local ou a atualização dos dados de uma pessoa. Nesta tabela ainda é possível observar a fonte de dado que deu origem à transformação, o tipo de situação até o momento, o instante em que a transformação ocorreu (ou o instante em que houve evolução na informação situacional) e o índice atual de confiança que a automação detém sobre a atual situação (certeza na situação).

O segundo ponto de vista é uma janela GIS baseado em mapa, com visualizações como sobreposições de acordo com a localização dos dados adquiridos geo-localizados. O uso de sobreposições em um mapa geo-referenciado é obrigatório para as operações de gerenciamento de cenários complexos de emergências, pois o domínio é dependente de localização para determinar o atendimento do evento de emergência. Cada objeto tem sua própria sobreposição, por exemplo, criminoso, objeto roubado, local do evento e vítimas. Cada sobreposição é acionável pela interação do utilizador para expor os atributos associados com o objeto. Quando um determinado objeto é visto na visualização do mapa, o objeto correspondente na tabela do objeto é realçado. O processo inverso também destaca a sobreposição no mapa.

A terceira visão é um quadro para apoiar o grafo relacional. A escolha de sua utilização é justificada pela necessidade do conhecimento hierárquico sobre como a informação é construída sobre as situações e seus objetos. A situação é representada pelo nodo central, composta pela relação entre objetos e seus atributos com ramificações ou não. Os objetos podem ser identificados e não têm relações com nenhum outro, por isso, podem estar em hierarquias diferentes. Neste caso, mesmo quando a composição não compõe uma situação, a mesma deve ser representada.

O tamanho do nó representa na hierarquia a importância, sendo o maior nó a situação (ex: emergência). O nível seguinte representa o conjunto de objetos (entidades) identificados no processo de inferência (ex: pessoas, objeto roubado, local). As folhas dos nós de menor tamanho representam os atributos dos objetos (ex: pontos de referência, descrições, status). 
A utilização da estrutura do grafo, também permite que os dados sejam reorganizadas e ter sua hierarquia atualizada ao longo do processo de análise para implementar os refinamentos no processo. Sempre que ocorre uma mudança em uma das visões, é refletido nas demais, atualizando suas informações.

\section{B. Apresentando o Nível 2 de SAW diretamente}

O objetivo é apresentar as informações necessárias para um segundo nível de consciência diretamente para apoiar a compreensão como resultada do processamento mínimo, como um primeiro indício de uma situação que provavelmente está acontecendo. A ideia é apresentar alguns valores já calculados em vez de depender de cálculo especialista sobre o Nível 1 de dados de SAW..

Algumas situações (composta por objetos e atributos) podem ser calculadas a priori para reduzir o cálculo mental do especialista que opera o sistema. Por exemplo, a parte automatizada pode fazer a fusão de informações de vários objetos do tipo "localização", identificada nas informações adquiridas.

Assim, em vez de apresentar todas as informações de entrada separadamente, informações fundidas com baixa dimensionalidade e mais significado podem ser adotadas. Portanto, todos os eventos com a mesma localização, e outros atributos, como um carro ou uma espécie de arma, podem ser combinados em informações única e significativa.

Nos casos em que não ocorre a fusão automática, o operador pode executá-la em qualquer um dos três pontos de vista, arrastando uma representação para outra. Tal abordagem é conhecida como fusão de interface e é uma das abordagens de refinamento para ser ainda mais detalhados. Essa abordagem ajuda a evitar a confusão em caso de vários eventos simultâneos, mas negligências numa granularidade maior no nível de atributos.

\section{Apoio a Consciência da Informação Global}

A "imagem da situação" deve estar sempre disponível. SAW Global é uma visão geral de conjunto da situação em uma linguagem de alto nível e de acordo com os objetivos do especialista. Ao mesmo tempo, informações detalhadas sobre tais objetos devem estar disponíveis de todas as maneiras, se solicitado.

Na maioria dos sistemas de Avaliação da Situação, SAW global é sempre visível e pode ser crucial para determinar quais os objetivos que têm grandes prioridades. Para tal, o grafo e tabela do objeto podem ser expandidos e contratados em demanda para expor e ocultar a hierarquia de objetos que compõe uma relação, visualmente e textualmente.

Além disso, quando uma relação candidato for detectada, indicado pelo ser humano ou do sistema, a tabela e grafo do objeto estabelece uma nova ligação gráfica indicando uma provável relação, que pode ou não ser aceita pelo especialista. Por novas associações em qualquer um dos pontos de vista, os outros respondem à associação e reorganizar-se. Dentro da mesa do objeto, as linhas são agrupadas. No mapa, as sobreposições são sobrepostas e no grafo, uma nova hierarquia é composta.

\section{Filtro de Informação}

Para evitar a sobrecarga, devem ser filtradas informações não relacionadas à SAW. A interface deve apresentar apenas as informações cruciais para alcançar os objetivos em cada tarefa por cada momento. Para tanto, foi desenvolvido um filtro interativo. Como a informação é inferida pela fase de aquisição, a informação existente sobre qualquer um dos objetos pode ser omitida ou destacada para uma análise específica.

Esse filtro é útil para reduzir o espaço de busca e determinação candidatos à fusão por meio da análise visual. No entanto, SAW não ocorre instantaneamente. Os seres humanos levam certo tempo a se orientar em relação a situações e atributos críticos. A filtragem ruim pode comprometer a visibilidade e a dinâmica do sistema que muda as horas extraordinárias. Além disso, SAW global pode ser depredado e impede humano ser proativo.

\section{E. Identificação explícita da ausência de informações}

Os seres humanos lidam com a ausência de meta-informação como algo positivo. Se houver leituras positivas, eles acham que uma leitura perdida também é positiva quando, na verdade, elas podem ser extremamente conflitantes e imprecisas. Os seres humanos agem de forma diferente quando eles sabem se há probabilidades de algo dar errado. A ausência de informações é geralmente tratada como correto e confiável.

Existem duas variações do problema: a inexistência de perigos, quando a informação foi analisada e não há nenhuma ameaça; e não há perigos desconhecidos, quando há alguns lugares que não foram cobertos ou com limitações de sensores.

Além disso, o stress e a carga de trabalho podem levar as pessoas a não prestar atenção à falta de informação. Os seres humanos são dependentes visuais. Outros apenas sabem por causa da experiência. Em aplicações militares, as linhas tracejadas são utilizadas para representar o desconhecido. $\mathrm{Na}$ referida interface, os nós tem sua cor interna atualizada toda vez que a qualidade da informação é corrigida, pincipalmente devido à completude, apresentada como o principal problema de qualidade de informações neste domínio. Como é o caso de um atributo desconhecido, o índice de qualidade global (incerteza) é a cor corrigida.

A cor do nó central do grafo representa da certeza na situação (nível de confiança do sistema na informação como um todo), calculada em função das dimensões de completude e atualidade de todos os objetos. A cor dos nós do primeiro nível representa o nível de qualidade do local de cada objeto/entidade, calculada em função da completude e atualidade da informação do objeto em particular. A cor dos nodos do segundo nível acompanha a cor do primeiro nível.

\section{F. Apoio a Verificação de Confiabilidade da Informação}

As pessoas consideram a confiabilidade do sensor para apoiar e pesar as suas opiniões sobre as informações produzidas e apresentadas. Assim, eles podem se beneficiar se eles sabem que certas informações não são confiáveis.

Sabe-se que no contexto de uso de dados HUMINT como dados de entrada, há uma grande chance dos dados adquiridos apresentarem algum tipo de problema de qualidade, compondo 
assim uma limitada confiabilidade nas fontes de tais dados. Para inferir e representar a confiabilidade da fonte de dado, neste caso, foram adotados os índices de qualidade local que foram produzidas pela mesma, ou seja, fatores que contribuem para a formação da confiabilidade dos sensores (contextos de leitura de um sensor). Quanto melhor a qualidade da informação gerada, maior a confiabilidade da fonte de dados.

Embora a confiabilidade dos valores possa ser apresentada numericamente, os autores afirmam que o uso de níveis de luminância é aconselhado (mais claro para o mais confiável).

Assim, a interface do usuário mostra formas alternativas de representação da qualidade. Quanto menor a categorização da qualidade da informação, mais rápida é a decisão do humano operador (elevado, médio, baixo) e os mesmos tendem a aceitar melhor as taxas mais baixas. Os usos de dados numéricos, analógicos e em classificação tendem a gerar decisões mais lentas.

Para a referida interface, emprega-se o uso de cores e formas, representando graficamente as dimensões pertinentes ao domínio e a generalização certeza da situação. Quanto mais próximo o nó se aproxima da cor verde, maior é a qualidade dos dados. Quanto mais próxima do vermelho, pior é a qualidade dos dados.

Além disso, através da interação com os nós no grafo, sobreposições no mapa e informações na tabela do objeto, os índices de qualidade da informação que compõem são apresentados para ilustrar como tal incerteza foi inferida. Essa abordagem permite que especialistas para verificar local e índices globais de qualidade sob demanda.

\section{G. Representando Eventos Históricos para Acompanhar Evolução das Informações}

A interface do usuário apresenta acesso gráfico e interativo para informações históricas por uma linha do tempo. Para isso, uma régua do tempo com intervalos de tempo que indicam informações que são entregue s ao sistema foi implementada. Em nossa abordagem do sistema avaliação da situação, a situação é algo que evolui ao longo do tempo. Situações passadas também podem ser restauradas e referidas.

Por isso, os especialistas podem acessar um evento na história e exibir objetos de informação, atributos e situações sob demanda. Ao selecionar um evento histórico, os outros pontos de vista estão definidos para mostrar as informações do evento selecionado.

Assim, existe uma possibilidade de retornar no passado e também para monitorar eventos em tempo real, para além de ser capaz de avançar diretamente para um tempo específico. Como um lado negativo, há uma possível perda de foco sobre os acontecimentos atuais relevantes e confusão sobre a realidade dos acontecimentos.

\section{H. Suportar a atualização dos níveis de qualidade de dados e informações e o gerenciamento da incerteza}

À medida que os humanos operadores precisam avaliar a utilidade da informação situacional, deve haver uma maneira para que os mesmos consigam adequar tal informa informação às necessidades de suas tarefas e consequentemente à decisão que deve ser tomada. Neste contexto há a necessidade que a atuação do humano reflita também na qualidade da informação, que é inferida e representada pela automação.
Uma vez que a informação situacional foi anteriormente inferida, os atributos referentes à qualidade da informação são também medidos e representados na interface, juntamente com as informações qualificadas, na forma de sugestões (cues) visuais que indicam tal qualificação. Neste contexto, a qualidade de dados e informações não apenas ajudam os humanos operadores a estabelecer um nível de confiança que devem depositar nas informações representadas, mas também orientam os mesmos a buscar recursos para a melhoria da qualidade da informação, em complemento às atividades que a automação já desempenhou, mas que eventualmente podem não ter sido suficientes para despertar a confiança do humano e estimular o processo de SAW.

Cabe então ao humano operador concordar e confiar nas as partes de informação situacional produzidas pela automação ou discordar e rejeitar a informação situacional preliminar, e trabalhar em benefício da melhoria da qualidade da informação e da aquisição de SAW.

Em nossa abordagem, não há a possibilidade de ajustar diretamente a qualidade da informação que já foi inferida pela automação. Entretanto, há a oportunidade de ajuste do processo do modelo Quantify para que suas etapas atuem em favor da melhoria da qualidade da informação. Como a decisão de buscar melhorar a qualidade da informação é tomada pelo humano operador, cabe à interface orientada a SAW acomodar os meios computacionais para implementar os refinamentos no processo.

Em nossa abordagem, o humano especialista é habilitado a atuar e ativar entradas na etapa de Gestão do Processo de Fusão, responsáveis por especificar três tipos de refinamento das atividades do processo de fusão Quantify, sendo estes: Refinamento da Aquisição, Refinamento da Fusão e Refinamento do Conhecimento Situacional.

Pelo Refinamento da Aquisição, o especialista é capaz de atuar diretamente na fase de "Aquisição de Dados HUMINT" para requisitar novas fontes de informação, solicitar novas leituras dos diversos sensores e estabelecem novos parâmetros operacionais de busca de informação. Como resultado, são geradas novos insumos para os processos internos desta etapa em questão, ou seja, novos objetos, atributos e propriedades, desencadeando os demais processos que podem se beneficiar de uma nova aquisição (Avaliação da Qualidade de Dados e Informações e consequentemente a Fusão de Informações). Como todas as outras formas de refinamento, a obtenção de novas inferências demanda a atualização do conhecimento situacional e consequentemente tudo que se sabe sobre a situação. À medida que novos refinamentos ocorrem, mais rico se torna o conhecimento situacional.

O Refinamento da Fusão foi desenvolvido para permitir que especialistas determinem manualmente os parâmetros de fusão, em vez de depender do processo automático de integração imediatamente após a aquisição de informação, a qual combina automaticamente todos os objetos e atributos encontrados para reduzir a dimensão da informação. Trata-se de uma nova parametrização do processo de fusão de dados e informações com critérios de qualidade, operacionalizada pela interface orientada a SAW e implementada por filtros que determinam os sob quais parâmetros a fusão será considerada. Eventualmente, o parâmetro a ser considerado para a fusão ainda não é conhecido pelo processo, cabendo ao humano 
especialista introduzi-lo ao conhecimento situacional e determinar que o mesmo deva ser considerado para as próximas integrações.

Finalmente, a Gestão do Conhecimento é a contribuição manual que humanos especialistas podem realizar para o conhecimento situacional, enriquecendo-o ao longo do tempo com informações advindas de seu próprio conhecimento em virtude de sua experiência, expertise ou ainda originário de fontes de informações externas que não foram ainda consideradas no processo de avaliação de situações e na interface SAW do SFDs.

Desta maneira, é possível que o humano operador insira, atualize ou remova objetos e atributos que compõem o conhecimento situacional diretamente, em interação intuitiva com o grafo da situação atual.

Atuando diretamente no conhecimento situacional, informações inseridas, atualizadas ou removidas refletem nas demais etapas do processo Quantify e podem ser considerada para Avaliação, Fusão e novamente na Representação semântica e gráfica na interface orientada a SAW.

Desta maneira, o conhecimento situacional pode ser incrementalmente reestruturado ao longo do tempo. A informação pode ser corrigida e semanticamente readequada. Assim, as associações entre os objetos, feitas por outro processo podem também serem refeitas. Certa nuance sobre a sinergia de objetos e a relação entre eles no cenário só podem ser inseridas por seres humanos.

Com estes mecanismos de refinamento da informação situacional, a interface detém o gerenciamento de informações de todo o processo e pode ser considerada como uma workbench de colaboração onde ambos, sistema e o humano operador, que fornecem e transmitem informações como um conhecimento parcial que evolui com o tempo. Assim, toda vez que uma nova informação é fornecida por um desses atores, o outro é habilitado a processa-la como parte de um novo conhecimento situacional.

No domínio de gerenciamento de cenários complexos de emergências, a interface esta presente onde um humano operador observa, se orienta, decide o que fazer e, em seguida, toma algumas medidas, que pode ser tanto a solicitação de refinamento ou uma decisão específica de domínio. Em nossa abordagem, este é o processo interno no qual o sistema compartilha o conhecimento parcial gerado pelas outras etapas, e em seguida, recebe as entradas dos operadores de forma cíclica.

\section{DISCUSSÃO}

Durante todo o processo de Avaliação da Situação, a informação situacional é propagada, evolui e pode ser comprometida devido às transformações sofridas no decorrer do tempo. A qualidade da informação por sua vez deve ser avaliada em toda a evolução deste processo. Desta forma, a utilização de interfaces de usuários para auxiliar na gestão e controle da informação auxilia o processo de aquisição, manter e propagar SAW.

No processo de Avaliação da Qualidade de Dados e Informações, comentamos sobre o controle em relação à qualidade dos dados que tem por objetivo qualificar a situação. Este processo auxilia a representação da informação a ser controlada na interface, para que trabalhando em conjunto interface e avaliação da qualidade possam auxiliar o operador especialista a obter, manter e melhorar sua SAW.

A criação da gestão das representações através do controle de uma interface, é baseado no resultado de uma ontologia de qualidade de dados e informações aplicados ao domínio de gestão de informações de emergências, e neste caso aplicado a um domínio de roubo.

A UI, é responsável por permitir o controle da Representação do Conhecimento Situacional, onde mecanismos de representação da informação não interferem semanticamente na informação, mas permitem incrementar o conhecimento das situações em relação as fases do processo.

Ambas as informações tem por objetivo dar suporte a UI orientada a SAW, onde ocorre a gestão plena da informação situacional e dos processos que contribuem para a sua formação.

Sempre que ocorre uma nova inferência de informações, a visualização gerida pela interface é atualizada, apresentando ao usuário uma nova Representação do Conhecimento Situacional. Desta forma, o usuário operador tem a possibilidade de manusear e participar de maneira proativa em relação às informações da situação.

Neste contexto, a qualidade dos dados e informações, quantificada e representada graficamente promove o acompanhamento mais rico de tal evolução, estimulando a percepção e até mesmo a compreensão direta da informação situacional. Uma vez melhor orientado pelas questões referentes à qualidade, o humano é capaz de tomar melhores decisões quanto à necessidade de refinamento ou encerramento do processo de avaliação da situação.

Com a utilização da interface para auxílio a gestão da informação, a cada nova interação dos processos internos, é gerado um efeito de propagação e evolução do conhecimento situacional, o que reflete em todas as próximas etapas de avaliação. Com isto, o humano operador tem a oportunidade de influenciar sobre a composição da informação. Quando há interações diretas do operador no processo de gestão da interface, é possível permitir que fontes externas ao processo sejam consideradas e avaliadas no contexto, incorporando as informações em tempo real à situação apresentada.

Adicionalmente, é possível observar, que embora o modelo como um todo tenha esta característica, os processos internos devem necessariamente seguir uma ordem pré-determinada para a geração de entradas e saídas que contribuam para o abastecimento de demais processos internos.

Não é obrigatório que todos os processos internos sejam ativados para que o humano operador determine $o$ encerramento do ciclo de analise, ou seja, processos como a fusão propriamente dita, podem não ser necessários para atingir SAW.

Com as informações apresentadas, conclui-se que cedendo aos operadores dos sistemas de avaliação de situações críticas a oportunidade de ter mais poderes em relação à gestão do processo na interface, somado as intensificações do relacionamento do operador através da qualidade da informação para construir representações mais fiéis em relação às situações permitem promover a aquisição, manutenção e retomada de SAW, o qual não foi largamente explorado. 


\section{REFERÊNCIAS}

[1] Baumgartner, N., Gottesheim, W., Mitsch, S., Retschitzegger, W., \& Schwinger, W. (2010). BeAware! - situation awareness, the ontology-driven way. Data \& Knowledge Engineering, 69(11), 1181-1193.

[2] Llinas, J., Bowman, C., Rogova, G., Steinberg, A., Waltz, E., \& White, F. (2004). Revisiting the JDL data fusion model II. SPACE AND NAVAL WARFARE SYSTEMS COMMAND SAN DIEGO CA.

[3] Kokar, M. M., \& Endsley, M. R. (2012). Situation awareness and cognitive modeling. IEEE Intelligent Systems, (3), 91-96.

[4] Endsley, M. R. (2011). Designing for situation awareness: An approach to user-centered design. CRC Press, 2011. p. 396. Second Edition. 2. ed. Boca Raton.

[5] Endsley, M. R. (1999). Situation awareness and human error: Designing to support human performance. In Proceedings of the high consequence systems surety conference.

[6] Endsley, M. R. (1995). A taxonomy of situation awareness errors. Human factors in aviation operations, 287-292.

[7] Rodgers, M. D., Mogford, R. H., \& Strauch, B. (2000). Post hoc assessment of situation awareness in air traffic control incidents and major aircraft accidents. Situation awareness analysis and measurement, 73-112.

[8] Blasch, E. P., Lambert, D., Valin, P., Kokar, M. M., Llinas, J., Das, S., ... \& Shahbazian, E. (2012). High level information fusion (hlif): Survey of models, issues, and grand challenges. Aerospace and Electronic Systems Magazine, IEEE, 27(9), 4-20.

[9] Pang, A. T., Wittenbrink, C. M., \& Lodha, S. K. (1997). Approaches to uncertainty visualization. The Visual Computer, 13(8), 370-390.

[10] O'Brien, J. A. (2004). Sistemas de informação e as decisões gerenciais na era da internet. Saraiva.

[11] Wang, R. Y., \& Strong, D. M. (1996). Beyond accuracy: What data quality means to data consumers. Journal of management information systems, 5-33.

[12] Batini, C., Cappiello, C., Francalanci, C., \& Maurino, A. (2009). Methodologies for data quality assessment and improvement. ACM Computing Surveys (CSUR), 41(3), 16.

[13] Correa, C. D., Chan, Y. H., \& Ma, K. L. (2009, October). A framework for uncertainty-aware visual analytics. In Visual Analytics Science and Technology, 2009. VAST 2009. IEEE Symposium on (pp. 51-58). IEEE.

[14] Mecella, M., Scannapieco, M., Virgillito, A., Baldoni, R., Catarci, T., \& Batini, C. (2002). Managing data quality in cooperative information systems. In On the Move to Meaningful Internet Systems 2002: CoopIS, DOA, and ODBASE (pp. 486502). Springer Berlin Heidelberg.

[15] Gershon, N. (1998). Visualization of an imperfect world. Computer Graphics and Applications, IEEE, 18(4), 43-45.
[16] Ann M. Bisantz, (2013) Uncertainty Visualization and Related Techniques. The Oxford Handbook of Cognitive Engineering.

[17] Riveiro, M. "Evaluation of uncertainty visualization techniques for information fusion." Information Fusion, $200710^{\text {th }}$ International Conference on. IEEE, 2007.

[18] Riveiro, M. (2007, July). Evaluation of uncertainty visualization techniques for information fusion. In Information Fusion, 2007 10th International Conference on (pp. 1-8). IEEE.

[19] MacEachren, A. M., Robinson, A., Hopper, S., Gardner, S., Murray, R., Gahegan, M., \& Hetzler, E. (2005). Visualizing geospatial information uncertainty: What we know and what we need to know. Cartography and Geographic Information Science, 32(3), 139-160.

[20] Matheus, C. J., Kokar, M. M., \& Baclawski, K. (2003, July). A core ontology for situation awareness. In Proceedings of the Sixth International Conference on Information Fusion (Vol. 1, pp. 545-552).

[21] Rogova, G. L., \& Bosse, E. (2010, July). Information quality in information fusion. In Information Fusion (FUSION), 2010 13th Conference on (pp. 1-8). IEEE.

[22] Nwiabu, N., Allison, I., Holt, P., Lowit, P., \& Oyeneyin, B. (2012, March). User interface design for situation-aware decision support systems. In Cognitive Methods in Situation Awareness and Decision Support (CogSIMA), 2012 IEEE International Multi-Disciplinary Conference on (pp. 332-339). IEEE.

[23] Yu, S., Deng, L., \& Zhang, Y. (2009, August). Visualization user interface for decision support systems. In Hybrid Intelligent Systems, 2009. HIS'09. Ninth International Conference on (Vol. 1, pp. 63-66). IEEE.

[24] Onal, E., Craddock, C., Endsley, M. R., \& Chapman, A. FROM THEORY TO PRACTICE: HOW DESIGNING FOR SITUATION AWARENESS CAN TRANSFORM CONFUSING, OVERLOADED SHOVEL OPERATOR INTERFACES, REDUCE COSTS, AND INCREASE SAFETY.

[25] Chai, H., \& Du, Y. (2012, August). A framework of situation awareness based on event extraction and correlation for military decision support. In Mechatronics and Automation (ICMA), 2012 International Conference on (pp. 192-196). IEEE.

[26] Gómez, M. A., Jiménez, S., Navarro, R., Lago-Peñas, C., \& Sampaio, J. (2011). Effects of coaches' timeouts on basketball teams' offensive and defensive performances according to momentary differences in score and game period. European Journal of Sport Science, 11(5), 303-308.

[27] Feng, Y. H., Teng, T. H., \& Tan, A. H. (2009). Modelling situation awareness for Context-aware Decision Support. Expert Systems with Applications, 36(1), 455-463. 